\title{
Minimizing the scattering of a nonmagnetic cloak
}

Zhang, Jingjing; Luo, Yu; Mortensen, Asger

Published in:

Applied Physics Letters

Link to article, DOI:

$10.1063 / 1.3366726$

Publication date:

2010

Document Version

Publisher's PDF, also known as Version of record

Link back to DTU Orbit

Citation (APA):

Zhang, J., Luo, Y., \& Mortensen, A. (2010). Minimizing the scattering of a nonmagnetic cloak. Applied Physics Letters, 96(11), 113511. https://doi.org/10.1063/1.3366726

\section{General rights}

Copyright and moral rights for the publications made accessible in the public portal are retained by the authors and/or other copyright owners and it is a condition of accessing publications that users recognise and abide by the legal requirements associated with these rights.

- Users may download and print one copy of any publication from the public portal for the purpose of private study or research.

- You may not further distribute the material or use it for any profit-making activity or commercial gain

- You may freely distribute the URL identifying the publication in the public portal

If you believe that this document breaches copyright please contact us providing details, and we will remove access to the work immediately and investigate your claim 


\title{
Minimizing the scattering of a nonmagnetic cloak
}

\author{
Jingjing Zhang, ${ }^{1, a)}$ Yu Luo, ${ }^{2}$ and Niels Asger Mortensen ${ }^{1}$ \\ ${ }^{1}$ Department of Photonics Engineering, DTU Fotonik, Technical University of Denmark, \\ DK-2800 Kongens Lyngby, Denmark \\ ${ }^{2}$ Department of Physics, The Blackett Laboratory, Imperial College London, London SW7 2AZ, \\ United Kingdom
}

(Received 16 December 2009; accepted 24 February 2010; published online 19 March 2010)

\begin{abstract}
Nonmagnetic cloak offers a feasible way to achieve invisibility at optical frequencies using materials with only electric responses. In this letter, we suggest an approximation of the ideal nonmagnetic cloak and quantitatively study its electromagnetic characteristics using a full-wave scattering theory. It is demonstrated that the forward scattering of the impedance matched cloak increases dramatically as the thickness of the cloak decreases. Nevertheless, it is still possible to effectively reduce the total scattering cross section with a very thin cloak whose impedance is not matched to the surrounding material at the outer boundary. Our analysis also provides the flexibility of reducing the scattering in an arbitrary direction. () 2010 American Institute of Physics. [doi:10.1063/1.3366726]
\end{abstract}

Realizing invisibility has been a dream of people for centuries. Recently, this topic has intrigued increasing interests, ${ }^{1-20}$ especially since the idea of designing invisibility cloaks with transformation methodology was proposed. ${ }^{1-3}$ Inspired by those theoretical studies, a cylindrical cloak at microwave frequency was soon experimentally realized, ${ }^{4}$ which was based on a set of simplified cloak parameters. ${ }^{5}$ However, as pointed out in Ref. 6, the design for the microwave cloak is not directly adaptable to optical frequencies where the concept of "invisibility" would have far reaching potential. Reference 6 gave a prescription for a nonmagnetic cloak working at the optical frequency range and an experiment on the optical cloak was later reported. ${ }^{7}$ The material parameters for those approximate nonmagnetic optical cloaks, originated from a linear transformation function, ${ }^{5}$ have a significant limitation that the impedance of the cloak is strongly mismatched at the outer surface. As an improvement approach, a high-order coordinate transformation, which renders the cloak perfectly matched to the free space, is made use of to eliminate the undesired scattering from the cylindrical nonmagnetic cloak. ${ }^{8,9}$ And Ref. 10 considers the boundary conditions at the inner boundary of the high-order point transformed cloak. However, it has been demonstrated theoretically that the drawback of the simplified cloak is more than just nonzero reflectance at the boundary. ${ }^{11}$ Several alternative approaches have been considered to overcome this obstacle. ${ }^{12,13}$

In this letter, we suggest a generalized form of simplified nonmagnetic cloak parameters, which can be optimized at a given frequency to minimize the scattering as desired. With a full wave analysis process, we theoretically demonstrate that the cloak system with a matching outer surface may have a large total scattering cross section when the cloak layer is kept thin, while it truly performs well for the backscattering detection. This method gives the freedom of designing nonmagnetic cloaks with small thicknesses, which are nearly transparent for detections from arbitrary given directions.

\footnotetext{
a) Author to whom correspondence should be addressed. Electronic mail: jinz@fotonik.dtu.dk.
}

For a transverse-magnetic (TM) incidence, we can make an approximation for the two-dimensional ideal cloak while sustaining the important nonmagnetic feature as, $\varepsilon_{\rho \rho}$ $=[f(\rho) / \rho]^{2}, \varepsilon_{\varphi \varphi}=\left[f^{\prime}(\rho)\right]^{2}, \mu_{z z}=1$. Here $f(\rho)$ is an arbitrary function which satisfies $0 \leq f(\rho) \leq R_{2}$ and $R_{1} \leq \rho \leq R_{2}$. The corresponding wave equation becomes

$$
\frac{1}{\rho} \frac{\partial}{\partial \rho} \frac{\rho}{\left[f^{\prime}(\rho)\right]^{2}} \frac{\partial}{\partial \rho} H_{z}+\frac{1}{f^{2}(\rho)} \frac{\partial^{2}}{\partial \varphi^{2}} H_{z}+k_{0}^{2} H_{z}=0 .
$$

In order to make the material parameters amenable to analytic solution of Eq. (1) and the boundary conditions $f\left(R_{2}\right)$ $=R_{2}$ and $f\left(R_{1}\right)=0$, we restrict ourselves to a transformation function expressed as

$$
f(\rho)=R_{2}\left(\frac{\rho^{2}-R_{1}^{2}}{R_{2}^{2}-R_{1}^{2}}\right)^{1 / 1+\alpha},
$$

where $\alpha$ is an adjustable parameter. The general solution of Eq. (1) takes the form $H_{z}=\left[k_{0} f(\rho)\right]^{1-\alpha / 2} B_{\nu}\left[k_{0} f(\rho)\right] e^{i n \varphi}$, where $k_{0}=\omega \sqrt{\mu_{0} \varepsilon_{0}}$, while $B_{\nu}$ represents a Bessel function of $\nu$ th order, where $\nu=\sqrt{n^{2}+(\alpha-1)^{2} / 4}$.

Suppose a TM polarized plane wave with the unit amplitude $H_{z}^{\mathrm{i}}=e^{i k_{0} x}$ is incident upon the cloak along the $x$ direction. The incident fields, scattered fields, and the fields inside the cloak layer are then conveniently written in polar coordination as

$$
\begin{aligned}
H_{z}^{\mathrm{i}}= & \sum_{n=-\infty}^{\infty} a_{n} J_{n}\left(k_{0} \rho\right) \cos n \varphi, \\
H_{z}^{\mathrm{s}}= & \sum_{n=-\infty}^{\infty} b_{n} H_{n}^{(1)}\left(k_{0} \rho\right) \cos n \varphi, \\
H_{z}^{\mathrm{c}}= & {\left[k_{0} f(\rho)\right]^{1-\alpha / 2} \sum_{n=-\infty}^{\infty}\left\{c_{n} J_{\nu}\left[k_{0} f(\rho)\right]\right.} \\
& \left.+d_{n} N_{\nu}\left[k_{0} f(\rho)\right]\right\} \cos n \varphi .
\end{aligned}
$$

Here $a_{n}=i^{n}, b_{n}, c_{n}$, and $d_{n}$ are all unknown expansion coefficients to be determined. By applying the continuities of $H_{z}$ 




FIG. 1. (Color online) Normalized total scattering cross sections in terms of $R_{2} / R_{1}$, where $R_{1}$ is fixed to be $1.5 \mu \mathrm{m}$. The insets show the field distributions for the cases where $R_{2}=4 / 3 R_{1}$ and $R_{2}=3 R_{1}$.

and $E_{\varphi}$ at $R_{2}$ and $R_{1}$ [The inner boundary of the cloak is assumed to be a perfect electric conductor (PEC)] and considering Eq. (2), all the undetermined coefficients can then be obtained as

$$
\begin{aligned}
& b_{n}=\frac{B J_{n}\left(k_{0} R_{2}\right)-A J_{n}^{\prime}\left(k_{0} R_{2}\right)}{A H_{n}^{(1) \prime}\left(k_{0} R_{2}\right)-B H_{n}^{(1)}\left(k_{0} R_{2}\right)} a_{n}, \\
& c_{n}=\frac{J_{n}\left(k_{0} R_{2}\right) H_{n}^{(1) \prime}\left(k_{0} R_{2}\right)-J_{n}^{\prime}\left(k_{0} R_{2}\right) H_{n}^{(1)}\left(k_{0} R_{2}\right)}{A H_{n}^{(1) \prime}\left(k_{0} R_{2}\right)-B H_{n}^{(1)}\left(k_{0} R_{2}\right)} a_{n}, \\
& d_{n}=0,
\end{aligned}
$$

where $\quad A=\left(k_{0} R_{2}\right)^{1-\alpha / 2} J_{\nu}\left(k_{0} R_{2}\right) \quad$ and $\quad B=\left(k_{0} R_{2}\right)^{-1+\alpha / 2}[(1$ $\left.-\alpha) J_{\nu}\left(k_{0} R_{2}\right) / 2+k_{0} R_{2} J_{\nu}^{\prime}\left(k_{0} R_{2}\right)\right] . J_{n}, N_{n}$, and $H_{n}^{(1)}$ represent the $n$th order of Bessel function, Neumann function, and Hankel function of the first kind, respectively. We notice that $b_{\mathrm{n}}$ is in general nonzero, indicating the existence of scattering outside the cloak.

In what follows, we aim to discuss the way of finding the value of the variable $\alpha$ which can optimize the performance of the cloak. As is commonly known, the impedance matching is a necessary condition for the reflection reduction. Hence we first take into account the condition that the impedance of the cloak is matched to the free space at the outer boundary $\left(\left.\varepsilon_{\varphi}\right|_{\rho=R_{2}}=\left[f^{\prime}\left(R_{2}\right)\right]^{2}=1\right)$. With Eq. (3), we straightforwardly have $\alpha=\left(R_{2}^{2}+R_{1}^{2}\right) /\left(R_{2}^{2}-R_{1}^{2}\right)$.

In order to study the performance of this cloak in terms of the thickness of the coating layer, we fix the inner boundary of the cloak to be $R_{1}=1.5 \mu \mathrm{m}$, and plot the normalized total cross section ${ }^{21}$ as a function of the outer boundary, as shown in Fig. 1. The source works at $200 \mathrm{THz}$ in all the calculations. The total scattering cross section increases dramatically as the thickness of the cloak decreases. The simplified cloak shows the effectiveness of reducing the scattering only when $R_{2} \gg R_{1}$. The insets display the electric field distributions for two cloaks where $R_{2}=3 R_{1}$ and $R_{2}=4 / 3 R_{1}$. It can be clearly observed that when $R_{2}$ is three times larger than $R_{1}$, the simplified cloak shows a good performance comparable to that of an ideal cloak. However, a shadow is formed in the forward path of the cloak when the outer radius is close to the inner radius.

Although the impedance matching condition provides a solution to the determination of parameters if the thickness of the cloak is not a significant factor, it is still necessary to find a general optimization method which is applicable to a cloak with thin coating layer. Here we study this problem by fixing both the inner and outer boundaries of the cloak and calculating the scattering cross sections in different direc-

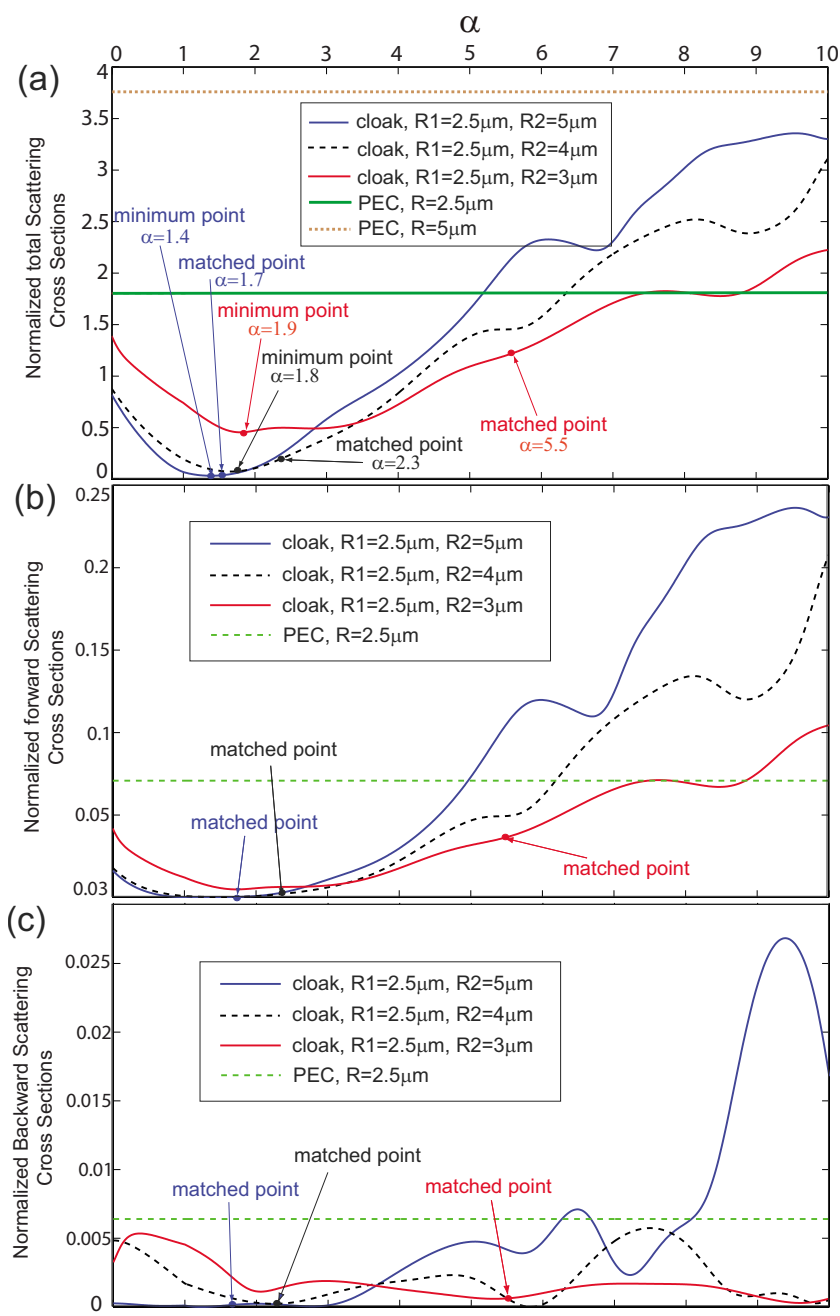

FIG. 2. (Color online) (a) Normalized total scattering cross sections in terms of $\alpha$ for cloaks of different thicknesses and PEC scatters of different radii. (b) Normalized forward scattering cross sections for cloaks of different thicknesses and the PEC scatterer. (c) Normalized backward scattering cross sections for cloaks of different thicknesses and the PEC scatterer.

tions in terms of $\alpha$. We consider cloaks with three different thicknesses (case 1: $R_{2}=5 \mu \mathrm{m}$; case 2: $R_{2}=4 \mu \mathrm{m}$; case 3: $R_{2}=3 \mu \mathrm{m} ; R_{1}=2.5 \mu \mathrm{m}$ for all cases) and calculate the normalized total scattering cross sections [see Fig. 2(a)]. By comparing the three curves, we find that as the thickness of the cloak reduces, the $\alpha$ which leads to a minimum total scattering cross section deviates more and more from the $\alpha$ which makes the simplified cloak matched to the free space. The curves also imply the way of minimizing the total scattering cross section. As a reference, we also plot the normal total scattering cross sections for the PEC scatterers with radii $R=2.5$ and $5 \mu \mathrm{m}$. It is worth noticing that when we choose certain values of $\alpha$, the corresponding cloaks themselves will induce such large scattering that the total scattering cross sections are even larger than that of the PEC core. For comparison, we also calculate the normalized forward scattering cross sections ${ }^{20}$ in terms of $\alpha$ to study the bistatic (transmitter and receiver in different locations) scattering properties of cloaks with different thicknesses.

Sometimes, for a monostatic detection where the transmitter and the receiver lie in the same position, we only need to consider the backscattering property of the cloak. In order to determine the optimized parameters in this condition, we plot the normalized backward scattering cross section in 
(a)
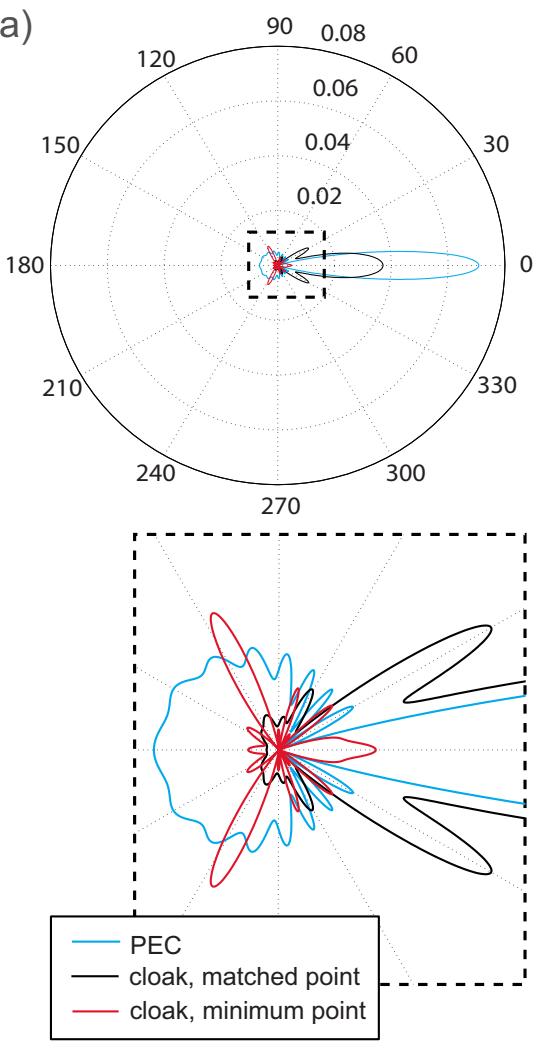

(b)

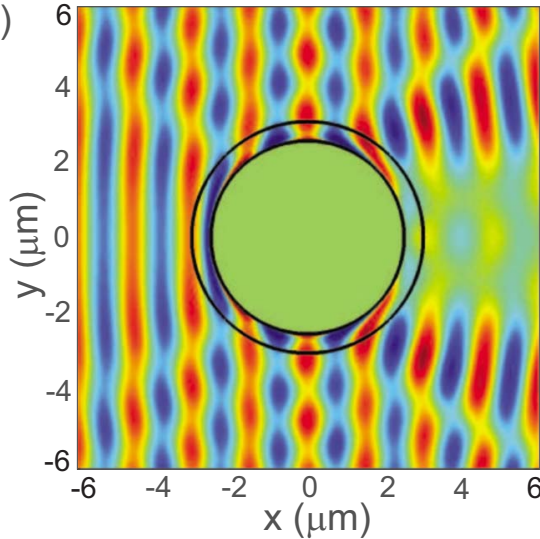

(c)

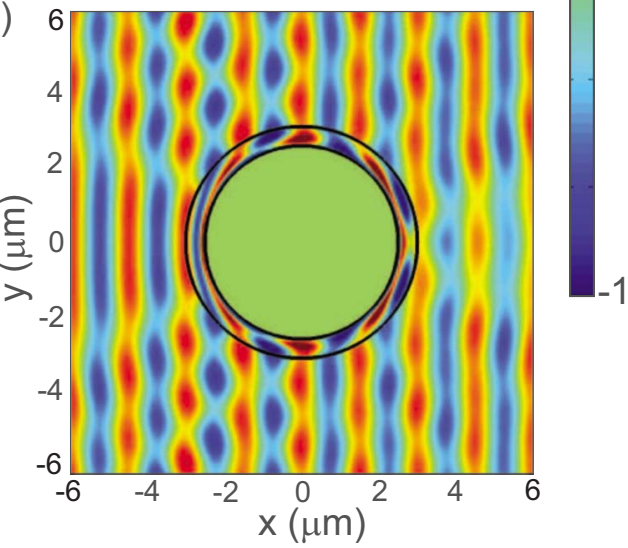

FIG. 3. (Color online) (a) Far field scattering patterns for two cloaks $\left(R_{1}=2.5 \mu \mathrm{m}, R_{2}=3 \mu \mathrm{m}\right)$, one with a matched outer boundary and the other with the minimum total scattering cross section. The scattering pattern of a PEC scatter with the radius of $2.5 \mu \mathrm{m}$ is also plotted as a reference. Panels (b) and (c) show the near field properties for the aforementioned two cloak cases, respectively. terms of $\alpha$, as shown in Fig. 2(c). For different thickness cases, we find that the cloaks with matching outer boundaries are almost invisible for monostatic detections.

In fact, for detector located in any other direction, we can plot the scattering cross section curve similarly and identify a certain $\alpha$ which can minimize the scattering. For the purpose of a clearer illustration, we plot the scattering pattern of a cloak whose outer and inner boundaries are $3 \mu \mathrm{m}$ and $2.5 \mu \mathrm{m}$, respectively. The cases for the $\alpha$ picked at the matched point and the $\alpha$ which makes a minimum total scattering cross section can be observed. Although the impedance matched cloak shows satisfying performance in a wide range of degrees in the backward side, it will induce a strong scattering in the forward directions, which is even larger than that caused by the PEC obstacle. The near field distributions for the corresponding matched cloak $(\alpha=5.5)$ and cloak with minimum total scattering cross section $(\alpha=1.8)$ are displayed in panels (b) and (c), respectively. By comparing Figs. 3(b) and 3(c), we can see that the forward scattering reduction property can be distinctly improved by choosing the $\alpha$ corresponding to the "minimum point" other than the "matched point."

In conclusion, we have analytically studied the performance of nonmagnetic cloaks with reduced material parameters. It is demonstrated that although the boundary impedance matching condition can render a cloak with good backscattering performance, it does not provide a recipe for reducing scattering in other orientations. As a solution, a parameter optimization scheme is suggested, which makes it possible to design cloaks of required scattering capability. Our method provides the flexibility of designing nonmagnetic cloaks for both monostatic and bistatic detections.
The work is supported by the Hans Christian Ørsted postdoctoral fellowship. J.Z. would like to appreciate the discussions with Professor Hongsheng Chen.

${ }^{1}$ J. B. Pendry, D. Schurig, and D. R. Smith, Science 312, 1780 (2006).

${ }^{2}$ U. Leonhardt, Science 312, 1777 (2006).

${ }^{3}$ A. Greenleaf, M. Lassas, and G. Uhlmann, Physiol. Meas 24, 413 (2003).

${ }^{4}$ D. Schurig, J. J. Mock, B. J. Justice, S. A. Cummer, J. B. Pendry, A. F. Starr, and D. R. Smith, Science 314, 977 (2006).

${ }^{5}$ S. A. Cummer, B.-I. Popa, D. Schurig, D. R. Smith, and J. B. Pendry, Phys. Rev. E 74, 036621 (2006).

${ }^{6}$ W. Cai, U. K. Chettiar, A. V. Kildishev, and V. M. Shalaev, Nat. Photonics 1, 224 (2007).

${ }^{7}$ I. I. Smolyaninov, Y. J. Hung, and C. C. Davis, Opt. Lett. 33, 1342 (2008).

${ }^{8}$ W. Cai, U. K. Chettiar, A. V. Kildishev, V. M. Shalaev, and G. W. Milton, Appl. Phys. Lett. 91, 111105 (2007).

${ }^{9}$ R. Weder, J. Phys. A: Math. Theor. 41, 065207 (2008).

${ }^{10}$ R. Weder, J. Phys. A: Math. Theor. 41, 415401 (2008).

${ }^{11}$ M. Yan, Z. Ruan, and M. Qiu, Phys. Rev. Lett. 99, 233901 (2007)

${ }^{12}$ Y. Luo, J. Zhang, H. Chen, S. Xi, and B.-I. Wu, Appl. Phys. Lett. 93, 033504 (2008).

${ }^{13}$ G. Castaldi, I. Gallina, and V. Galdi, Phys. Rev. B 80, 125116 (2009).

${ }^{14}$ F. Zolla, S. Guenneau, A. Nicolet, and J. B. Pendry, Opt. Lett. 32, 1069 (2007).

${ }^{15}$ M. Yan, Z. Ruan, and M. Qiu, Opt. Express 15, 17772 (2007).

${ }^{16}$ J. Zhang, J. Huangfu, Y. Luo, H. Chen, J. A. Kong, and B.-I. Wu, Phys. Rev. B 77, 035116 (2008).

${ }^{17}$ Y. Luo, H. Chen, J. Zhang, L. Ran, and J. A. Kong, Phys. Rev. B 77, 125127 (2008).

${ }^{18}$ R. Liu, C. Ji, J. J. Mock, J. Y. Chin, T. J. Cui, and D. R. Smith, Science 323, 366 (2009).

${ }^{19}$ H. Chen, Z. Liang, P. Yao, X. Jiang, H. Ma, and C. T. Chan, Phys. Rev. B 76, 241104 (2007).

${ }^{20}$ D.-H. Kwon and D. H. Werner, Appl. Phys. Lett. 92, 013505 (2008)

${ }^{21}$ C. A. Balanis, Advanced Engineering Electromagnetics (Wiley, New York, 1989). 